\title{
Implant Files, Medizinprodukterecht und Patientensicherheit: Antworten der Politik
}

\section{MATTHIAS DETTLOFF}

Dr. Matthias Dettloff ist Referent für

Methodenbewertung beim GKV-Spitzenverband, Berlin

\section{Im November 2018 veröffentlichte ein}

Journalistenkonsortium die sogenannten „Implant

Files", in denen zahlreiche Medizinprodukteskandale der Vergangenheit dargestellt sind. Die zentrale Botschaft: Das geltende Medizinprodukterecht ist intransparent, schützt Patienten nicht ausreichend und lädt zu Missbrauch ein, da kaum aussagekräftige klinische Studien durchgeführt werden müssen. Es ist zu hoffen, dass die neue europäische Medizinprodukteverordnung in ein paar Jahren die Lage verbessern wird. Bis dahin müssen jedoch andere Instrumente greifen. Im Folgenden wird erörtert, ob das geplante Implantateregistergesetz und die beabsichtigten Änderungen der Bundesregierung an der Nutzenbewertung von Hochrisikomedizinprodukten die Probleme lösen - oder verschärfen.

\section{Medizinprodukterecht in Europa - Zeit des Umbruchs}

\subsection{Medizinprodukteskandale und der Kampf um neue Spielregeln}

Die Verkehrsfähigkeit von Medizinprodukten unterliegt dem EU-Recht. Aktuell gelten noch verschiedene Richtlinien, die in den neunziger Jahren des letzten Jahrhunderts in Kraft getreten sind und das Inverkehrbringen von Medizinprodukten, aktiven Implantaten und In-vitroDiagnostika regeln. Die Defizite dieses Rechtsrahmens wurden auch durch die Veröffentlichungen rund um die „Implant Files“ nochmals deutlich dargestellt. Ein wesentlicher Kritikpunkt an den alten Spielregeln ist neben der völlig fehlenden Transparenz des Marktgeschehens (niemand hat z. B. einen Überblick darüber, wie viele Brustimplantate von wie vielen Herstellern tatsächlich europaweit auf dem Markt sind), dass Medizinprodukte von Herstellern vor ihrem Inverkehrbringen kaum mit hochwertigen und aussagekräftigen klinischen Studien geprüft werden müssen und dass es auch keine behördliche Zulassung gibt. Stattdessen erklären die Hersteller eigenverantwortlich die Konformität ihrer Produkte mit dem europäischen Recht. Abhängig von der Risikoklasse seines Produkts (Abbildung 1) beauftragt ein Hersteller dabei eine privatwirtschaftlich organisierte Benannte Stelle mit der Prüfung des eigenen Qualitätsmanagementsystems sowie der Produkte. Die letztere Prüfung erfolgt abhängig von der Risikoklasse in unterschiedlichem Ausmaß. Der Hersteller kann seine Benannte Stelle europaweit frei wählen. Hersteller müssen vorab eine klinische Bewertung ihrer Produkte vorgenom- 


\section{Abbildung 1: Klassifikation von Medizinprodukten}

Medizinprodukte sollen helfen, eine Krankheit zu lindern oder zu heilen. Sie werden entweder äußerlich am Menschen oder im Körperinneren angewendet. Im Gegensatz zu Arzneimitteln wirken Medizinprodukte primär physikalisch. Um die immense Produktvielfalt zu systematisieren, werden sie gemäß der EU-Richtlinie für Medizinprodukte (93/42 EWG) einzelnen Risikoklassen zugeordnet.

\begin{tabular}{|c|c|}
\hline Klasse I & $\begin{array}{l}\text { Produkte und Gebrauchsartikel zur äußeren Anwendung (nicht invasiv) mit geringem Gefährdungspotenzial } \\
\text { und niedrigem Risiko. } \\
\text { Beispiele: Heftpflaster, Mundspatel. }\end{array}$ \\
\hline Klasse Ila & $\begin{array}{l}\text { Produkte, die kurzzeitig (weniger als } 30 \text { Tage) invasiv angewendet werden oder von denen aus anderen } \\
\text { Gründen ein mittleres Risiko ausgeht. } \\
\text { Beispiele: Geräte zum diagnostischen Ultraschall, Infusionskanülen. }\end{array}$ \\
\hline Klasse Ilb & $\begin{array}{l}\text { Produkte mit erhöhtem Risiko z. B. aufgrund einer dauerhaften chirurgisch-invasiven Anwendung, einer } \\
\text { entfalteten biologischen Wirkung oder der Abgabe von Energie in Form von ionisierender Strahlung. } \\
\text { Ebenso Produkte, deren Anwendung unter Berücksichtigung der Art der betreffenden Stoffe, des } \\
\text { betreffenden Körperteils und der Art der Anwendung eine potentielle Gefährdung darstellt } \\
\text { Beispiele: Röntgengeräte, externe Defibrillatoren, Überwachungsmonitore sowie alle Implantate außer } \\
\text { denen, die ausdrücklich in die Risikoklasse III fallen. }\end{array}$ \\
\hline Klasse III & $\begin{array}{l}\text { Produkte mit hohem Risiko, z. B. solche, die mit dem zentralen Kreislaufsystem, dem zentralen } \\
\text { Nervensystem oder den Herzen in Berührung kommen oder die ein Medikament zum Bestandteil haben. } \\
\text { Beispiele: Koronarstents, Prothesen der Bauchaorta. Bestimmte Produktgruppen sind per EU-Richtlinie } \\
\text { zusätzlich in die Klasse III eingeordnet worden: Brustimplantate sowie Knie- und Hüftendoprothesen }\end{array}$ \\
\hline $\begin{array}{l}\text { Aktive implantierbare } \\
\text { medizinische Geräte }\end{array}$ & $\begin{array}{l}\text { Produkte mit hohem Risiko, die jedoch durch eine eigene EU-Richtlinie (90/385 EWG) geregelt sind. } \\
\text { Produktklasse entspricht hinsichtlich der gesetzlichen Bestimmungen der Klasse III. } \\
\text { Beispiele: implantierbare Herzschrittmacher, Defibrillatoren und Neurostimulatoren. }\end{array}$ \\
\hline
\end{tabular}

Quelle: Wolf et al. 2017

men haben. Grundlage dieser Bewertung können alle möglichen publizierten und nicht publizierten klinischen Daten sein. Vielfach reicht ein Bezug auf bereits in den Verkehr gebrachte Produkte von Wettbewerbern, die „gleichartig“ zum eigenen Produkt sind. Da die Intensität der eigentlichen Produktprüfung von der Risikoklasse abhängt, wird nur bei Klasse III-Produkten und aktiven Implantaten immer eine Prüfung der klinischen Bewertung durch eine Benannte Stelle vorgenommen. Bei Produkten der Klasse IIa und IIb ist dies nur für ein repräsentatives Produkt einer Produktkategorie bzw. einer Produktgruppe erforderlich. Hat beispielsweise ein Hersteller mehrere verschiedene Stents für Beinarterien im Programm, muss er nur für ein Produkt die entsprechenden Unterlagen vorlegen. Ist die Prüfung erfolgreich, erhält der Hersteller ein entsprechendes CE-Zertifikat und kann damit alle zugehörigen Produkte, auch die nicht eigens geprüften, in den Verkehr bringen.

Im September 2012 veröffentlichte die EU-Kommission den Entwurf einer neuen Medizinprodukteverordnung. Sie verfolgte damit u. a. das Ziel, die Qualität der Arbeit der Benannten Stellen (damals gab es europaweit mehr als 80 Stellen, die von den Medizintechnikherstellern frei gewählt werden konnten, in intensivem Wettbewerb miteinander standen und offensichtlich auch unterschiedlich hohe Anforderungen an die Qualität der zu zertifizierenden Produkte stellten) zu vereinheitlichen. Was dann kam, war in der Intensität überraschend: Die politische Debatte des Entwurfs fand nämlich unter dem Eindruck mehrerer großer Medizinprodukteskandale statt, beispielsweise um die fehlerhaften Brustimplantate des Herstellers Poly Implant Prothèse, welche fehlerhafte Hüllen aufwiesen und darüber hinaus mit (für den Einsatz im Körper minderwertigem) Industriesilikon gefüllt waren, oder um Metall-auf-Metall-Hüftendoprothesen, die aufgrund Metallabriebs zu Knochennekrosen und erhöhten Kobaltwerten im Blut führten. Das europäische Parlament diskutierte in der Folge eine Verschärfung des Verordnungsvorschlags, die deutlich über die Vorstellungen der Kommission hinausging. So wurde beispielsweise von der Berichterstatterin des EU-Parlaments für diesen Verordnungsentwurf eine behördliche Zulassung für Hochrisikomedizinprodukte gefordert. Das rief die Gegenkräfte auf den Plan. Besonders die Verbände der Medizintechnik-Industrie arbeiteten massiv gegen die Initiative der Berichterstatterin an - auf eine Weise und in einem Ausmaß, das auch von erfahrenen Akteuren als weit über das „normale“ $\mathrm{Maß}$ von Lobbyismus hinausgehend cha- rakterisiert wurde (Implant Files 2018). Nach Darstellung in den „Implant Files“ kam besonders viel Widerstand gegen die zentrale Zulassung aus Deutschland (Implant Files 2018), das über eine sehr einflussreiche Medizintechnikbranche verfügt. Viele andere von der Industrie unabhängige Organisationen der Wissenschaft, des Verbraucherschutzes, der Sozialversicherungen und nicht zuletzt die GKV haben sich dagegen für eine Erhöhung der Transparenz und des Sicherungsniveaus der Bedingungen für die Anwendung von Medizinprodukten stark gemacht.

Am Ende einer hitzigen und chaotischen Auseinandersetzung wurden fast eintausend Änderungsanträge in die parlamentarische Debatte eingebracht, bevor das Parlament im Oktober 2013 beschloss, bei dem alten System der Benannten Stellen zu bleiben und lediglich ein paar zusätzliche Kontrollinstrumente einzuführen. Ein Kontrollinstrument stellt im Vergleich zum alten Recht eine deutliche Verschärfung dar: Demnach sollen die Benannten Stellen für bestimmte Hochrisikomedizinprodukte die vom Hersteller eingereichten Unterlagen sowie ihre eigene Bewertung an eine unabhängige Expertengruppe bei der EU-Kommission schicken, die zur vorgelegten Einschätzung Stellung nehmen und Empfehlungen abgeben kann. Die Benannte Stelle ist 
zwar nicht an die Empfehlungen dieser Expertengruppe gebunden, muss aber schriftlich begründen, warum sie ggf. davon abweicht. Die entsprechenden Dokumente sind zu veröffentlichen. Selbst diese abgeschwächte unabhängige Produktprüfung, die als „Scrutiny-Verfahren“ bezeichnet wird, wurde allerdings von den Herstellerverbänden der Medizintechnikindustrie energisch bekämpft. Sie sei innovationsfeindlich und würde die Markteinführung von neuen Produkten unnötig verzögern. In den nachfolgenden Abstimmungsprozessen im Rat der EU (in dem einzig die Bundesrepublik gegen den Vorschlag stimmte) sowie im nachfolgenden „Trilog“ zwischen Kommission, Rat und EU-Parlament setzte sich diese Regelung erfreulicher Weise - trotz Ablehnung der Bundesregierung - durch (Tebroke 2015).

\subsection{Die neue Verordnung: Verbesserung des Status quo - hoffentlich}

Die neue Medizinprodukteverordnung trat am 25. Mai 2017 in Kraft. Sie wird allerdings erst ab dem 26. Mai 2020 gelten und die alten Richtlinien ablösen. Die dreijährige Frist zwischen Inkrafttreten und tatsächlichem Geltungsbeginn, die jedenfalls eine genügend lange Übergangsfrist für die Industrie darstellen sollte, ist der Tatsache geschuldet, dass beispielsweise noch die neue EU-Datenbank EUDAMED aufgebaut werden muss, die Benannten Stellen Vorlauf für ihre Neubenennung nach den Vorgaben der Verordnung benötigen und auch weitere Regelungen und Infrastrukturen, wie z. B. die oben genannte unabhängige Expertengruppe für das «Scrutiny-Verfahren“, erst etabliert werden müssen. EU-Verordnungen müssen nicht in nationales Recht umgesetzt werden, sondern gelten anders als EU-Richtlinien in allen Mitgliedstaaten unmittelbar.

Auch wenn es nach wie vor keine behördliche Zulassung für Medizinprodukte gibt, sind einige verschärfende Regelungen in der Verordnung zu finden, die im Sinne der Patientensicherheit sehr begrüßenswert sind. Sie sind im Folgenden kurz zusammengefasst.

A. Klassifizierung von Medizinprodukten: Sämtliche Total- oder Teilprothe-

sen von Gelenken werden mit der neuen Verordnung in die höchste Risikoklasse III eingeordnet. Nach den alten Regeln gehörten bisher nur Endoprothesen des Knie-, Hüft- oder Schultergelenks der Klasse III an, alle übrigen Gelenkprothesen waren Produkte der Klasse IIb. Dasselbe gilt für Bandscheibenprothesen oder Wirbelkörperersatzprothesen sowie für chirurgische Netze. Auch diese Produkte werden nun anders als bisher in die höchste Risikoklasse III einsortiert. Aktive implantierbare medizinische Geräte, deren Verkehrsfähigkeit bisher über eine eigene Richtlinie geregelt wurde, werden nun in die Risikoklasse III eingeordnet.

B. Benannte Stellen: Bevor ein Mitgliedstaat eine Konformitätsbewertungsstelle benennen kann, muss ein dreiköpfiges Bewertungsteam, bestehend aus Sachverständigen von zwei anderen Mitgliedstaaten sowie einem Sachverständigen der EU-Kommission, die Bewerbungsunterlagen der Stelle bewerten sowie eine Vor-Ort-Begehung durchführen um zu prüfen, ob die Benannte Stelle die Verordnungsvorgaben hinsichtlich Infrastruktur und Personalausstattung erfüllt. Die zuständigen Überwachungsbehörden der Mitgliedstaaten müssen regelmäßig nach einem bestimmten Prüfschema die Arbeit der von ihnen Benannten Stellen auditieren. Für implantierbare Produkte der Klasse III sowie

Viel Widerstand gegen die zentrale Zulassung kam aus Deutschland, das über eine sehr einflussreiche Medizintechnikbranche verfügt.

für aktive Medizinprodukte der Klasse IIb, die Medikamente abgeben, gilt das angesprochene Scrutiny-Verfahren, nach dem die Benannte Stelle die unabhängige Expertengruppe der EU-Kommission konsultieren und ihr die Produktunterlagen sowie ihre eigene Bewertung zur Stellungnahme vorlegen muss.

C. Klinische Bewertungen: Die neue Verordnung schreibt vor, dass künftig nur in Ausnahmefällen von einer klinischen Prüfung abgesehen werden darf - nämlich dann, wenn es sich um die Weiterentwicklung eines Produktes desselben Herstellers handelt, dessen Gleichartigkeit mit dem ursprünglichen Produkt nachgewiesen werden kann. Wenn sich der Hersteller auf das Produkt eines Wettbewerbers bezieht, kann er lediglich dann auf eine klinische Prüfung verzichten, wenn er einen vollständigen Zugriff auf die Produktdokumentation des Wettbewerbers hat, was nur in wenigen Ausnahmen der Fall sein dürfte. Bei der klinischen Bewertung muss der Hersteller zwar nach wie vor die „Leistung“ seines Medizinproduktes bewerten, allerdings ist hier auch eine Bewertung des klinischen Nutzens anhand patientenrelevanter Endpunkte einzubeziehen.

D. Zweckbestimmung von Medizinprodukten: „Zweckbestimmung“ bezeichnet die Verwendung, für die ein Produkt entsprechend den Angaben des Herstellers bestimmt ist. Nach den alten EU-Richtlinien konnte der Hersteller die Zweckbestimmung seines Medizinproduktes relativ frei festlegen. Beispielsweise gibt es derzeit Stentprothesen auf dem Markt, die für die Versorgung von verengten Oberschenkel- und Leistenarterien klinisch geprüft wurden, deren Zweckbestimmung allerdings einen weiter gefassten Einsatz in „peripheren Gefäßen“ vorsah. Also auch in anderen als den genannten Arterien sowie auch in peripher gelegenen Venen; darüber hinaus nicht nur bei Gefäßverengungen, sondern auch bei Gefäßerweiterungen (Aneurysmen). Die EU-Verordnung schreibt nun vor, dass die Zweckbestimmung im Einklang mit der klinischen Bewertung stehen und auch bei Produkten der Klasse IIb und III auf dem CE-Zertifikat der Benannten Stelle angegeben sein muss.

E. Transparenz: Die neue Verordnung sieht vor, dass eine europäische Datenbank für Medizinprodukte (EUDAMED) etabliert wird, die wenigstens in Teilen öffentlich zugänglich ist. Auch wenn beklagenswerter Weise der öffentliche Zugang beschränkt ist, wird sich dadurch gleichwohl die Transparenz deutlich verbessern. Denn erstens ermöglicht diese Datenbank erstmals, sich einen Überblick über alle in den Verkehr gebrachten Medizinprodukte zu verschaffen. Zweitens werden für implantierbare Produkte sowie alle Produkte der Klasse III sogenannte „Kurzberichte über Sicherheit und klinische Leistung “ öffentlich zugänglich gemacht. Ebenso müssen von den Herstellern Angaben zur Zweckbestimmung sowie sämtliche 
Prüfberichte zu klinischen Prüfungen auch zu vorzeitig abgebrochenen - in der Datenbank veröffentlicht werden. All diese Informationen sind derzeit nur schwer recherchierbar.

Der Veröffentlichungszeitpunkt der „Implant Files“ lenkt den Blick auf eine schwierige und unglückliche Situation. Die neue Verordnung ist zwar seit über einem Jahr in Kraft. Allerdings werden die ersten Medizinprodukte frühestens ab Mai 2020 nach den neuen Regelungen in den Verkehr gebracht. Es gelten bis dahin nach wie vor die alten Medizinprodukterichtlinien und die nationalen Gesetze. Alle bisherigen, von den „Implant Files“ beschriebenen Probleme und Skandale, die möglicherweise in den kommenden zwei Jahren auftreten und aufgedeckt werden, konnten und können daher naturgemäß von den Verschärfungen der neuen Verordnung nicht verhindert werden. Da bisher noch kein einziger Zertifizierungsprozess nach den neuen Regeln durchlaufen und auch die Datenbank EUDAMED noch nicht entwickelt ist, vermag auch niemand genau zu sagen, ob die in Kraft getretenen Regeln tatsächlich die ihnen zugeschriebenen Wirkungen entfalten werden - oder ob sie, wie von einigen befürchtet, ins Leere laufen.

Gegen diese Befürchtung, dass die Verordnung keinerlei Effekt haben wird, sprechen jedoch die Bestrebungen seitens der Industrie, die Medizinprodukteverordnung nachträglich zu verändern und aufzuweichen. Angeblich würden die kurzen Übergangsfristen und ein erkennbarer zeitlicher Verzug bei der Implementierung der Verordnung dazu führen, dass viele Hersteller ihre Produkte vom Markt nehmen müssten. Daher müssten die Übergangsfristen verlängert werden. Zudem sei die Regelung in der vorliegenden Form innovationsfeindlich und müsse geändert werden (Fraunhofer ITEM 2018). Mit diesen Forderungen, die von einigen EU-Parlamentariern übernommen wurden (Meißner 2018), musste sich auch die EU-Kommission beschäftigen, die sich zu einem Statement genötigt sah, dass die Implementierung der Verordnung im Zeitplan sei und keine zusätzlichen Maßnahmen oder Veränderungen notwendig seien (Politico 2018). Die Veröffentlichung der „Implant Files" hat hoffentlich einen Beitrag dazu geleistet, dass eine Verzögerung des Geltungsbeginns politisch nicht weiter erwogen wird.

\section{Deutsches Implantateregister: die Lösung aller Probleme?}

\subsection{Haltbarkeit und \\ Rückverfolgbarkeit von Implantaten}

Bundesgesundheitsminister Jens Spahn reagierte Ende November vergangenen Jahres auf die Veröffentlichung der „Implant Files" damit, dass er die Etablierung eines nationalen Implantateregisters ankündigte: „Wir wollen wissen, welchem Patienten welches Produkt eingebaut wird. Und wir wollen nachprüfen können, wie lange diese halten."1 Der Referentenentwurf für ein neues Gesetz wurde für Anfang Januar 2019 angekündigt.

Die Idee eines Implantateregisters ist nicht neu. Politische Bestrebungen, ein solches Register per Gesetz zu beschließen, gibt es seit dem Koalitionsvertrag aus dem Jahr 2013 (Koalitionsvertrag 2013). Sogenannte Endoprothesenregister existieren in verschiedenen Ländern schon länger, in Schweden und Finnland beispielsweise seit ziemlich genau vierzig Jahren. Auch in Deutschland gibt es seit vielen Jahren verschiedene freiwillige Initiativen für Implantationsregister, die vor allem auf Initiative medizinischer Fachgesellschaften etabliert wurden. Beispiele dafür sind das Herzschrittmacher- und Defibrillatoren-Register, das seit den achtziger Jahren existiert, das Aortenklappenregister oder das deutsche Endoprothesenregister EPRD. Besonders die beiden letztgenannten Beispiele zeigen jedoch die Limitationen dieser Bestrebungen auf: da die Meldung an das Register jeweils freiwillig erfolgt, werden nicht alle Implantationen gemeldet. Dies schmälert die Aussagekraft eines solchen Registers deutlich, denn wenn nicht alle Fälle zwingend gemeldet werden müssen, könnten gerade die Fälle, bei denen es Probleme gab, durch Nichtmeldung dem Register verloren gehen.

Aus diesem Grund sind grundsätzliche politische Forderungen an ein Implantateregister zu stellen: Es muss eine Verpflichtung geben, ausnahmslos alle Fälle zu melden und auszuwerten und es muss gewährleistet sein, dass man bei erkannter Auffälligkeit einzelner Implantate, die einen Produktrückruf oder ärztliche Untersuchungen erforderlich machen, die betroffenen Patientinnen und Patienten auch zügig identifizieren und kon- taktieren kann. Das oben genannte Zitat des Gesundheitsministers verdeutlicht, dass das neu zu etablierende deutsche Implantateregister die Erreichbarkeit genau dieser Ziele sicherstellen soll.

Allerdings gibt es eine ganze Reihe von Fragen, die durch gesetzliche Regelungen geklärt werden müssen: Wie lässt sich eine Meldung von Implantationen an das Register durch Krankenhäuser unbürokratisch und datensparsam realisieren? Wie lässt sich eine eindeutige Identifikation von verbauten Implantaten sicherstellen? Wie lässt sich prüfen, dass tatsächlich alle Implantationen von Krankenhäusern an das Register gemeldet worden sind und dass alle verbauten Implantate auch im Register geführt werden? Wie lässt sich die millionenfache Übermittlung von Implantationsdaten realisieren, ohne dass $\mathrm{Pa}$ tientinnen und Patienten vorab einwilligen müssen - was andernfalls einen immensen bürokratischen Aufwand bedeuten und die Idee einer Vollerhebung konterkarieren würde? Wie lassen sich ggf. ambulant vorgenommene Implantationen im Register abbilden und überprüfen? Was ist mit Implantationen, die von Gesunden ,in Eigenleistung" bezahlt werden und nicht von Krankenversicherungen übernommen werden, z. B. von Brustimplantaten? Wie lässt sich die Behandlung von Privatversicherten im Register erfassen? Und wie ist sichergestellt, dass Patientinnen und Patienten auch Jahrzehnte nach erfolgter Operation noch zuverlässig kontaktiert werden können?

\subsection{Notwendige Schritte zur Umsetzung}

Aus Sicht der GKV sind die folgenden Schritte notwendig:

A. Krankenkassen-Abrechnungsdaten einbeziehen: Werden Versicherte der GKV im Krankenhaus mit Implantaten versorgt, so übermittelt die Einrichtung entsprechende Abrechnungsdaten an

1 https://rp-online.de/politik/deutschland/ spahn-verspricht-mehr-transparenz-undpatientensicherheit-bei-medizinprodukten aid-34723693. Zugriff am 3.1.2018. 
die Krankenkasse, die bestimmte prozedurenbezogene Angaben enthalten. Die zuständige Krankenkasse prüft die Abrechnungsdaten auf Plausibilität und lässt gegebenenfalls durch den medizinischen Dienst der Krankenkassen eine detailliertere Prüfung vornehmen. Aus diesem Grund sollte das Implantateregister zur Plausibilitätsprüfung von Implantations-Krankenhausmeldungen die Abrechnungsdaten der Krankenkassen heranziehen.

B. Abrechnungsdaten der Krankenhäuser mit Implantate-Identifikationsnummern versehen: Bisher erfahren die Krankenkassen nicht, mit welchen Implantaten ihre Versicherten versorgt wurden. Die Abrechnungsdaten geben nur wieder, dass ein Implantat eingesetzt wurde. Es ist notwendig, die Abrechnungsdaten um Implantate-Identifikationsnummern $\mathrm{zu}$ erweitern, um sicherzustellen, dass nur im Register geführte Implantate von den Kassen bezahlt werden. Außerdem können die Krankenkassen vom Rückruf betroffene Versicherte besser unterstützen.

C. Patientenkontakte über Kassen organisieren: Bisher sind die Krankenhäuser dafür verantwortlich, bei bestimmten Implantateklassen im Falle eines Produktrückrufs oder bei notwendig werdenden medizinischen Maßnahmen die betroffenen Patientinnen oder Patienten zu kontaktieren. Die Erfahrung zeigt jedoch, dass diese Regelung impraktikabel ist, da Krankenhäuser in der Regel nicht erfahren, wenn behandelte Personen nach mehreren Jahren ihren Wohnort wechseln. Sie erfahren oftmals auch nicht, wenn eine Person sich in einer anderen Einrichtung erneut operieren lässt. Die zuständige Krankenkasse hat jedoch alle notwendigen Informationen, um betroffene Versicherte im Falle einer Auffälligkeit ihres Implantats umgehend kontaktieren zu können und z. B. die Vorstellung in einer medizinischen Einrichtung zu empfehlen.

\subsection{Was ein Implantateregister nicht leistet}

Es ist offenkundig, dass Implantateregister nur ein Instrument der Marktüberwachung und Produktkontrolle sowie gegebenenfalls der Qualitätssicherung sein können. Aussagen zu Nutzen und Risiken der Produkte und der Implantation erlauben Register nicht. Deshalb kann auch das nationale Implantateregister keine Nutzenbewertung ersetzen oder Skandale mit
Implantaten verhindern, wenn diese auf unzulänglichen Erkenntnissen beim Inverkehrbringen basieren. Es kann lediglich dafür sorgen, dass fehlerhafte Implantate früher als bisher identifiziert und weitere Implantationen dieser Produkte schneller als bisher verhindert werden können.

\section{Hochrisikomedizinprodukte im Krankenhaus: Ist alles Neue auch nützlich?}

Die von den „Implant Files“ dargestellten regulatorischen Defizite der geltenden EU-Richtlinien für Medizinprodukte und aktive implantierbare medizinische Geräte haben auch auf nationaler Ebene Ausdruck gefunden. Im Dezember 2013 hatten die damaligen Koalitionspartner in ihrem Koalitionsvertrag das politische Ziel formuliert: „Krankenhäuser, in denen neue Medizinprodukte mit hoher Risikoklasse zum Einsatz kommen, sollen verpflichtet werden, sich in der Phase nach der Markteinführung an Nutzen- und Sicherheitsstudien des G-BA [gemeint ist der Gemeinsame Bundesausschuss] zu beteiligen“ (Koalitionsvertrag 2013).

Die GKV hatte zu diesem Zeitpunkt entsprechende Regelungen bereits mehrmals nachdrücklich gefordert und eigene Konzepte entwickelt (GKV-Spitzenverband 2010, GKV-Spitzenverband 2014), wie es erreicht werden kann, dass Innovationen unter kontrollierten Bedingungen und verbunden mit der Durchführung von qualitativ hochwertigen klinischen Studien eingeführt werden können. Die Vorgabe des Koalitionsvertrags wurde vom Gesetzgeber im Jahr 2015 im sogenannten „Versorgungsstärkungsgesetz“ umgesetzt (Bundestag 2015). Die Neuregelung beschränkt sich dabei allerdings auf Methoden unter Einsatz von solchen Hochrisikomedizinprodukten, die deutliche Mehrkosten verursachen, weshalb Krankenhäuser für deren Anwendung Entgelte für neue Untersuchungs- und Behandlungsmethoden, sogenannte NUBEntgelte, beantragen. Der Einsatz der Medizinprodukte im Rahmen der Methode muss außerdem einen „besonders invasiven Charakter" aufweisen, was aufgrund der beschlossenen Rechtsdefinitionen $z$. B. nur sehr wenige Produkte der Risikoklasse IIb erfüllen. Schließlich muss das Vorgehen ein „neues theoretisch-wissenschaftliches Konzept" aufweisen.
Das Verfahren sieht vor, dass der G-BA von Krankenhäusern detaillierte Informationen über neue Methoden erhält, für die erstmalig NUB-Entgelte angefragt wurden. Die Informationen sollen auch Angaben über Studienergebnisse mit den entsprechenden Medizinprodukten enthalten. Innerhalb von drei Monaten nach Informationsübermittlung durch Krankenhäuser hat der G-BA auf Basis der übermittelten Informationen einen Beschluss zu fassen. Für den Beschluss gibt es drei Möglichkeiten: Der patientenrelevante Nutzen der Methode kann erstens als belegt angesehen werden - in diesem Fall kann die Methode ohne weitere Prüfung erbracht werden. Der Nutzen der Methode kann zweitens noch nicht nachgewiesen sein, jedoch das „Potenzial einer erforder-
Ein Implantateregister kann lediglich dafür sorgen, dass fehlerhafte Implantate früher als bisher identifiziert werden.

lichen Behandlungsalternative" aufweisen. In diesem Fall beschließt der G-BA eine Erprobungsrichtlinie, nach der Krankenhäuser, welche die Methode anwenden wollen, sich an einer klinischen Studie beteiligen müssen, die dem Nutzennachweis dient. Bietet die Methode nach den vorliegenden Erkenntnissen nicht das Potenzial einer erforderlichen Behandlungsalternative, leitet der G-BA Verfahren ein, an dessen Ende das Verbot der Methode stehen soll - folgerichtig, insofern ein fehlendes Potenzial es als nicht akzeptabel erscheinen lässt, die Methode anzuwenden und auch keine hinreichenden Voraussetzungen für die weitere Evaluation zeigt.

Allerdings zeigte sich in der Praxis schnell, dass der Gesetzgeber bei der Ausgestaltung zu sehr darauf bedacht war sicherzustellen, dass möglichst wenige Methoden mit Hochrisikomedizinprodukten von der Regelung betroffen sind und vor allem betroffene Produkte in ihrer Anwendung möglichst nicht „behindert" werden und ihre Finanzierung durch die GKV auch außerhalb von Studien gewährleistet sein sollte (Dettloff et al. 2016, Wolf et al. 2017). Insbesondere sollte sichergestellt werden, dass für eine Anwendung von Methoden im Krankenhaus generell nicht erforderlich ist, dass deren patientenrele- 
vanter Nutzen belegt ist. Vielmehr sollte bereits das Vorliegen des „Potenzials einer erforderlichen Behandlungsalternative“, also die Möglichkeit, dass die Methode nach vorliegenden Daten einen patientenrelevanten Nutzen haben könnte, für eine generelle Anwendbarkeit ausreichen. Dieser Rechtsauffassung hat das Bundessozialgericht allerdings in zwei wegweisenden Urteilen widersprochen und festgestellt, dass das Vorliegen eines Potenzials einer erforderlichen Behandlungsalternative nicht ausreicht, damit eine Methode das Qualitätsgebot der gesetzlichen Krankenversicherung erfüllt. Damit steht das dem Gesetz zugrundliegende Konzept, Leistungen auch ohne vorliegenden Nutzenbeleg in der allgemeinen Krankenhausversorgung zu akzeptieren, in Frage (Bundessozialgericht 2017, Bundessozialgericht 2018).

Der G-BA hatte im Jahr 2017 einige Bewertungen von insgesamt zwei Hochrisikomedizinprodukten in verschiedenen Indikationen durchgeführt und dabei mehrfach festgestellt, dass kein Potenzial einer erforderlichen Behandlungsalternative vorliegt, was offenbar eine politisch nicht gewollte Entscheidung darstellte. (GKV-Spitzenverband 2018).

Daher ist es kaum verwunderlich, dass die Bundesregierung auf die aktuelle Rechtsprechung und die vorliegenden Bewertungsergebnisse des G-BA reagiert hat. Im vorliegenden Gesetzesentwurf (als Teil des Termin-Service und Versorgungsgesetz TSVG) werden einige grundlegende Änderungen bei der Bewertung von Methoden mit Medizinprodukten hoher Risikoklasse vorgenommen (Bundesgesundheitsministerium 2018). Allerdings weisen diese in die falsche Richtung. Nachfolgend die beiden geplanten besonders fatalen Änderungen für die Patientensicherheit:

A. Wegfall der Potenzialbewertung: Nach den vorliegenden Änderungen soll der G-BA künftig nicht mehr bewerten, ob die Anwendung eines Hochrisikomedizinprodukts das Potenzial einer erforderlichen Behandlungsalternative aufweist. Stattdessen soll es künftig für die Anwendung dieser Methoden ausreichen, wenn der G-BA bei seiner Bewertung feststellt, dass es keinen eindeutigen Nachweis gibt, dass sie unwirksam oder schädlich ist - und diese schlichte Feststellung (die auch immer schon dann erfüllt ist, wenn überhaupt keine Studien vorliegen) die Methode zu Lasten der Kassen anwendbar machen soll.

B. Wegfall der Teilnahmeverpflichtung der Krankenhäuser an Erprobungs- studien: Gleichzeitig soll zudem die Verpflichtung der die Methode anwendenden Krankenhäuser entfallen, sich an Studien des G-BA zu beteiligen - die zwar nach wie vor durch den G-BA zu entwickeln wären, aber aufgrund der gestrichenen Teilnahmeverpflichtung wohl nie zustande kommen würden.

Das Ziel, die Erkenntnislage für riskante Methoden und die ihnen zugrunde liegenden Medizinprodukte zu verbessern, spielt nach dieser Regelung offensichtlich keine Rolle mehr. Wenn das Gesetz in dieser Form beschlossen wird, ist das ursprüngliche Ziel, Methoden mit Medizinprodukten hoher Risikoklasse vor ihrer flächendeckenden Anwendung kontrolliert zu erproben und Erkenntnisse darüber zu erlangen, welche Patienten tatsächlich von der Methode profitieren und welchen sie vielleicht sogar schadet, offiziell zu Grabe getragen.

\section{Fazit}

Die „Implant Files“ haben über Medizinprodukteskandale berichtet, die in den letzten Jahren vorgefallen sind. Wie die akribische Arbeit zeigt, sind es erstaunlich und erschreckend viele. Betroffen sind vielfältige Produktklassen wie Brustimplantate, künstliche Bandscheiben, Defibrillatorelektroden, sondenlose Herzschrittmacher, Hüftendoprothesen. Dies zeigt zugleich, dass es in Bezug auf diese Produkte und die medizinische Versorgung, bei der diese zum Einsatz kommen, nicht nur zufällig und punktuell Probleme gibt, sondern dass diese systematisch und strukturell bedingt sind. Die vom G-BA durchgeführten Bewertungen von Hochrisikomedizinprodukten bestätigen diesen Befund: Sie sollten mit so wenigen klinischen Daten in die Versorgung eingeführt werden, dass der G-BA nicht einmal ein „Potenzial einer erforderlichen Behandlungsalternative“ bescheinigen konnte. Zwar steigt der Handlungsdruck auf die Politik, die Rahmenbedingungen zu verändern, immer dann, wenn die öffentliche Aufmerksamkeit nach einem Skandal groß ist. Dies war bei der Diskussion um die europäischen Gesetzesreformen der Fall, die immerhin einige Verbesserungen bringen dürfte. Dies ist auch jetzt der Fall. Dabei ist es insbesondere das Verdienst derjenigen die die „Implant Files“ zusammengetragen haben, gleichsam von außen ermittelt und die systemischen Schwachstellen deutlich gemacht zu haben. Eine durchgreifende Verbesserung ist nicht von einer einzelnen, durchaus sinnvollen, Maßnahme wie dem Implantateregister zu erwarten, die man ohnehin geplant hatte und die man dann adhoc schützend vor sich hält. Dazu sind weitergehende Maßnahmen notwendig. Es ist zu hoffen, dass die Änderungen des Rechtsrahmens auf europäischer Ebene dazu führen, dass neue Medizinprodukte vor ihrer Markteinführung künftig besser geprüft werden und hier auch schon die Grundlage für eine effektive Nutzenbewertung gelegt wird. Es ist ferner zu hoffen, dass das deutsche Implantateregister künftig einen Beitrag dazu leistet, fehleranfällige Produkte früher als bisher zu identifizieren und gezielt gut organisierte Maßnahmen zu ergreifen. Für die Versorgung ist zu hoffen, dass Bundesregierung und Gesetzgeber erkennen, dass eine ungeprüfte flächendeckende Anwendung von Hochrisikomedizinprodukten, bevor ausreichende Erkenntnisse über ihre Sicherheit, ihre Wirksamkeit und ihren patientenrelevanten Nutzen vorliegen, der falsche Weg ist - insbesondere weil aktuelle Gesetzgebungsvorschläge darauf hinauslaufen, die gerade erst begonnene Nutzenbewertung von Hochrisikoprodukten faktisch wieder einzustellen. Die Erkenntnisse aus den Implant Files erfordern genau das Gegenteil! Eine weitgehend ungeprüfte flächendeckende Anwendung

\section{Es ist zu hoffen, dass Bundesregierung und Gesetzgeber erkennen, dass eine ungeprüfte Anwendung von Hochrisikomedizinprodukten der falsche Weg ist.}

im Krankenhaus weiter zu ermöglichen, ist auch für Hersteller der schlechtere Weg, wenn sich erst im Nachhinein zeigt, dass ihr Produkt die schlechtere Behandlungsalternative darstellt. Vorschläge, wie man die Versorgung besser und innovationsfreundlicher gestalten kann, liegen auf dem Tisch (GKV-Spitzenverband 2017, Dettloff et al. 2016, Wolf et al. 2017). Wir sind bereit, das Unsere zu tun, um diesen Weg zu beschreiten. 


\title{
Literatur
}

Bundesgesundheitsministerium (2018).

Entwurf eines Gesetzes für schnellere Termine und bessere Versorgung (Terminserviceund Versorgungsgesetz - TSVG). https:// www.bundesgesundheitsministerium.de/ fileadmin/Dateien/3 Downloads/Gesetze und_Verordnungen/GuV/T/Kabinettvorlage Gesetzesentwurf TSVG.pdf, [Abruf am 09.01.2019].

Bundessozialgericht (2017). Revisionsurtei vom 19.12.2017 zu einem Urteil des Landessozialgerichts Baden-Württemberg vom 23.11.2016. Aktenzeichen B 1 KR 17/17 R

Bundessozialgericht (2018). Revisionsurteil vom 24.02.2018 zu einem Urteil des Landessozialgerichts Baden-Württemberg vom 31.08.2016. Aktenzeichen B 1 KR 10/17 R.

Bundestag (2015). Gesetz zur Stärkung der Versorgung in der gesetzlichen Krankenversicherung (GKV-Versorgungsstärkungsgesetz GKV-VSG). 16. Juli 2015. Bundesgesetzblatt Jahrgang 2015 Teil I Nr. 30, Bonn, 22. Juli 2015.

Dettloff $M$, Wolf K, Egger B (2016). Innovationen zum Nutzen der Patientinnen und Patienten. GKV 90 Prozent, Ausgabe 03, Dezember 2016 https://www.gkv-90prozent.de/ ausgabe/03/autorenbeitrag/03_innovationenmedizinprodukte/03 innovationenmedizinprodukte.html, [Abruf am 09.01.2019]. Fraunhofer ITEM (2018). Medizintechnik: Europäische Medizinprodukteverordnung bremst Innovationsstandort Deutschland. https:// www.item.fraunhofer.de/de/presse-medien/ presseinformationen/pm-medical-deviceregulation.html, [Abruf am 09.01.2019].

GKV-Spitzenverband (2010). Innovationszentren in der GKV-Versorgung - Patientenwohl als Wettbewerbsvorteil. https://www. gkv-spitzenverband.de/media/dokumente/ presse/pressemitteilungen/2010/Konzept Innovationszentren Internet30112010_15101.pdf, [Abruf am 09.01.2019].

GKV-Spitzenverband (2014). Medizintechnische Innovationen im Krankenhaus: Nutzen- und Sicherheitsstudien - Vorschlag zur Umsetzung des Koalitionsvertrages. https://www. gkv-spitzenverband.de/media/dokumente/ presse/publikationen/Positionspapier Medizintechnische_Innovationen_barrierefrei. pdf, [Abruf am 09.01.2019].

GKV-Spitzenverband (2017). Positionspapier des GKV-Spitzenverbandes für eine am Nutzen für die Versicherten orientierte Einführung von Innovationen in die medizinische Versorgung. https://www.gkv-spitzenverband.de/media/ dokumente/presse/presse themen/innovationszentren/Positionspapier_Innovationsbewertung_2017.pdf, [Abruf am 09.01.2019].

GKV-Spitzenverband (2018). BMG blockiert G-BA-Beschluss für mehr Patientensicherheit. GKV 90 Prozent, Ausgabe 09, Juni 2018. https://www.gkv-90prozent.de/ausgabe/09/ meldung/09 medizinprodukte/09 medizinprodukte.html, [Abruf am 09.01.2019].
Implant Files (2018). Die Angstmacher. https:// projekte.sueddeutsche.de/implantfiles/politik/ implant-files-die-lobbyisten-e547677/, [Abruf am 09.01.2019].

Koalitionsvertrag (2013). Deutschlands Zukunft gestalten. Koalitionsvertrag zwischen CDU, CSU und SPD. 18. Legislaturperiode. 13.12.2013, S. 57. https://www.cdu.de/sites/ default/files/media/dokumente/koalitionsvertrag.pdf, [Abruf am 09.01.2019].

Meißner (2018). Debatte mit der Kommission über Medizinprodukte: Brexit darf keinen Engpass für Patientenversorgung in der EU auslösen. https://www.gesine-meissner.de/ presse/pressemitteilungen/details/debatte-mitder-kommission-ueber-medizinprodukte-brexitdarf-keinen-engpass-fuer-patientenversorgungin-der-eu-ausloesen.html, [Abruf am 09.01.2019]

Politico (2018). Bienkowska: Notified Bodies could be approved this year for new device regs. https://www.politico.eu/pro/bienkowskanotified-bodies-could-be-approved-this-year-fornew-device-regs/, [Abruf am 09.01.2019].

Tebroke E (2015). Medizinprodukterecht - EU findet gemeinsame Linie. Pharmazeutische Zeitung 26, 2015. https://www.pharmazeutischezeitung.de/ausgabe-262015/eu-rat-findetgemeinsame-linie/, [Abruf am 09.01.2019]

Wolf K, Dettloff M, Egger B (2017). Hochrisikomedizinprodukte in der GKV: Was ist eine innovationsfreundliche Gesundheitspolitik? Gesundheit und Sozialpolitik 3-4: 75-83.

\section{Eine neue Würdekategorie für Menschen mit Demenz}

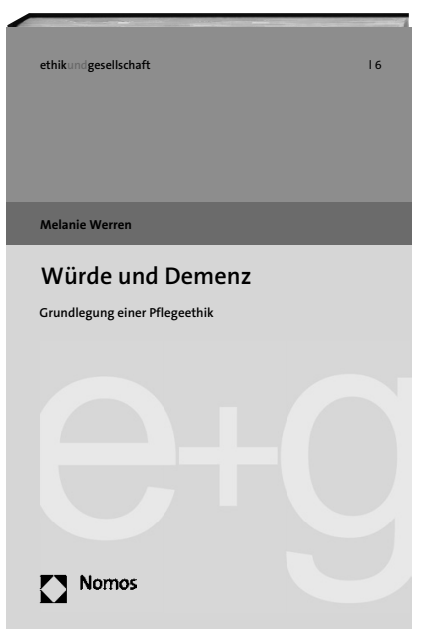

\author{
Würde und Demenz \\ Grundlegung einer Pflegeethik \\ Von Dr. Melanie Werren \\ 2019, 244 S., brosch., 46,- $€$ \\ ISBN 978-3-8487-5546-2 \\ (ethikundgesellschaft, Bd. 6) \\ nomos-shop.de/40601
}

In diesem Buch wird eine pflegeethische Grundlegung der Würdekategorie geboten - exemplarisch dargestellt anhand der Würde von Menschen mit Demenz. Einerseits wird ein für ihre Situation angemessener Würdebegriff entwickelt. Andererseits werden daraus praktische Konsequenzen abgeleitet.

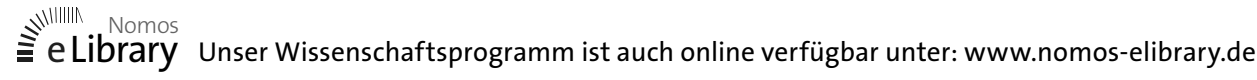

